\title{
A prospective study on comparison of cervical length measured transvaginally and bishop score in predicting successful labour induction
}

\author{
T. E. Balaji*, Vijaya S.
}

Department of Obstetrics and Gynaecology, Institute of Obstetrics and Gynaecology, Madras Medical College, Chennai, Tamil Nadu, India

Received: 18 January 2021

Revised: 14 February 2021

Accepted: 15 February 2021

\section{*Correspondence:}

Dr. T. E. Balaji,

E-mail: sruthiraghavbalaji@gmail.com

Copyright: (C) the author(s), publisher and licensee Medip Academy. This is an open-access article distributed under the terms of the Creative Commons Attribution Non-Commercial License, which permits unrestricted non-commercial use, distribution, and reproduction in any medium, provided the original work is properly cited.

\begin{abstract}
Background: Induction of labour is one of the common interventions in obstetric practice. Cervical assessment has been used as a prediction of the successful vaginal delivery. This study is designed to investigate transvaginal ultrasonographic cervical measurement as a predictor of duration of labour and successful induction resulting in vaginal delivery and compare the performance of ultrasonographic cervical measurement with that of the Bishop score in predicting the outcome of labour induction.

Methods: It is a prospective observational study. In this study, for the 100 primigravida women with gestational age of 37-42 weeks of gestation admitted for induction of labour, the cervical length was measured by transvaginal ultrasound and then Bishop Score was assessed by digital examination. Predictive values for successful labour induction were detected and compared.

Results: Using Spearman's rho correlation both TVS cervical length and Bishop score have significant correlation in predicting the success of induction of labour. Cervical length is the better predictor of the likelihood of delivering vaginally within $24 \mathrm{hrs}$. In the receiver operating characteristic curves, the best cut-off points for the prediction of successful induction was $26 \mathrm{~mm}$ for cervical length and 4 for the Bishop Score. However, TVS cervical length appears to be a better predictor than the Bishop Score, with a sensitivity of $58.1 \%$ and a specificity of $100 \%$ compared to $70.3 \%$ and $45.5 \%$ respectively.

Conclusions: Transvaginal sonographic measurement of cervical length is a better predictor of the likelihood of successful induction of labour within $24 \mathrm{hrs}$ of induction and increased vaginal deliveries within 48 hours of induction when compared to Bishop Score.
\end{abstract}

Keywords: Bishop score, Cervical length, Induction of labour, Transvaginal ultrasound

\section{INTRODUCTION}

Induction of labour is done before the spontaneous onset of labour by medical or surgical methods in approximately $20 \%$ of pregnancies. ${ }^{1}$ Cervical assessment by Bishop score has been used to assess the cervix and predict the likelihood of successful vaginal delivery. Bishop originally observed that nulliparous women undergoing induction of labour with a cervical score $>8$ had the same likelihood of vaginal delivery as did women in spontaneous labour. ${ }^{2}$ Induction of labour with low cervical score has been associated with failed induction, prolonged labour, dysfunctional labour, instrumental deliveries and a high rate of caesarean section.

The transvaginal ultrasonographic measurement of the cervix represent a more accurate assessment of the cervix than cervical assessment by Bishop score. The 
advantages of the transvaginal ultrasonographic assessment is quantitative, easily reproducible and with minimal discomfort to the patient. The limitations of cervical assessment in closed cervix are difficulty in assessing the length of supravaginal portion of the cervix and assessment of the effacement which starts at the internal os.

This study was done to determine if transvaginal ultrasonographic measurement of cervical length could predict the outcome of induction better than clinical assessment obtained by the Bishop score. If so, transvaginal ultrasonographic measurement of cervical length can be used as a predictor for successful vaginal deliveries and shorter duration of labour following induction.

\section{METHODS}

The study was a Prospective Observational study conducted at the Institute of Obstetrics and Gynaecology, Madras Medical College, Chennai. The study was conducted for the period of about one year from March 2019 to March 2020.About 100 primi gravida with gestational age ranging between $37-42$ weeks, who were admitted for labour induction under Obstetrics and Gynaecology Department were included in the study after considering the exclusion and inclusion criteria. The inclusion criteria for the study population were nulliparous patients, singleton pregnancy, live foetus with vertex presentation, intact amniotic membranes, gestational age between 37-42 weeks, reassuring NST pattern before induction, no contraindications for vaginal delivery, patients who are willing to give consent for the study and Bishop's score $\leq 6$.The exclusion criteria were vaginal bleeding, allergic to prostaglandins, patients in active phase of labour, history of uterine surgery like previous LSCS, myomectomy, presence of severe maternal or foetal compromise such as severe preeclampsia, severe IUGR, cardiac disease etc.

\section{Method of collection of data}

The selected patients baseline characters such as age, gestational age at induction and indication for induction were noted. After informed consent is obtained, Transvaginal ultrasonographic measurement of cervical length is performed with the standard longitudinal view of the cervix while the patient's bladder is empty. GE VOLUSON 730 PRO TVS Probe IC5-9 H instruments with $5-9 \mathrm{MHz}$ is using to measure the cervical length. Cervical length is measured by keeping the probe $3 \mathrm{~cm}$ away from the posterior fornix. The cervical length is defined as the length between the internal and external OS. After sonography the Bishop Score is determined by the digital examination by the resident responsible for the induction. The residents were masked to the cervical length measurement. Induction of labour was carried out according to the standard protocol of our hospital. Prostaglandin E2 gel was inserted into the cervical canal within 1 hour of cervical assessment. The patient was reassessed after 6 hours. If she did not exhibit regular uterine contractions and cervical change, a second dose of PG E2 is administrated intracervically. Maximum of 3 doses can be repeated. Subsequent dose is withheld if,

The patient is in active labour

Rupture of membrane

\section{If cervical effacement $>60 \%$ and $\mathrm{OS} \geq 3 \mathrm{~cm}$}

Regular uterine contractions 2-3 in 10 minutes.

Augmentation of labour was done as per labour room protocol.Successful induction of labour is defined as active labour occurring at the end of induction protocol (12 hrs from the last dose).Failed induction is defined as an inability to achieve the active phase of labour corresponding to cervical dilatation of $\geq 3 \mathrm{~cm}$ within 12 hours from the last dose of PG E2.Failure to progress is defined as no cervical dilation during the active phase of labour for the last 2 hours or no descent of the foetus head during the second stage of labour for at least 1 hour despite adequate uterine contractions. This is considered as an indication for caesarean delivery for failure to progress. The Primary outcome measures assessed were the Induction to delivery interval $<24 \mathrm{hrs}$ and the Secondary outcome measures assessed were the Induction - Active phase interval $<12 \mathrm{hrs}$ and the number of vaginal deliveries $<48 \mathrm{hrs}$.

\section{Statistical analysis}

The software used for statistical analysis was IBM Statistical package for the social sciences SPSS-20.Chisquare test was used to investigate whether distribution of categorical variables differ from one another and $p$ value less than or equal to 0.05 was considered significant. For the continuous variables Mean and Standard deviation were calculated. ROC curve analysis was conducted to determine the optimal cut off points of Bishop score and transvaginal USG cervical length for successful vaginal deliveries with 24 hours.

\section{RESULTS}

The variables of the hundred primigravida women enrolled in the study such as age distribution, indication for induction were analysed. The age distribution was analysed with mode of delivery, Bishop score and Cervical length by TVS ultrasound and concluded that there was no significant association (Table 1).

Among the hundred women included in the study the indication for induction of labour, $23 \%$ were induced for Post-dated pregnancy $18 \%$ for Gestational hypertension, $50 \%$ for prolonged latent phase of labour, $7 \%$ for decreased AFI $<8 \mathrm{~cm}, 2 \%$ for decreased foetal movements. Among the study group, $4 \%$ women had a 
Bishop score of $1,7 \%$ had score of $2,17 \%$ had score of $3,20 \%$ had score of 4 and 26 had score of 5 . Women with transvaginal cervical length $<2 \mathrm{~cm}$ were $12 \%, 31 \%$ of women were with $2.1-2.5 \mathrm{~cm}$ and $57 \%$ were with $>=2.6 \mathrm{~cm}$. Vaginal delivery occurred in $85 \%$ of women within 48 hours and $74 \%$ of these, delivery was within 24 hours of induction, about $55 \%$ had $<12$ hours of induction to active phase interval. The analysis of Bishop score showed that there was no significant difference for women whose delivery interval was within 24 hours and more than 24 hours, but cervical length showed significant difference i.e. If cervical length is more than the period of induction delivery interval will be increased (Table 2).

Table 1: Comparison of age wise distribution among mode of delivery, bishop score and cervical length.

\begin{tabular}{|c|c|c|c|c|c|c|}
\hline Age & & $15-20$ years & 21-25 years & 26-30 years & 31-35 years & \multirow{3}{*}{$\begin{array}{l}\text { Chi square } 7.090 \\
p>0.05(0.07) \text { non } \\
\text { significant }\end{array}$} \\
\hline \multirow{2}{*}{$\begin{array}{l}\text { Mode of } \\
\text { delivery }\end{array}$} & Vaginal & $31(37.6 \%)$ & $40(47.1 \%)$ & $12(14.1 \%)$ & $1(1.2 \%)$ & \\
\hline & LSCS & $1(6.7 \%)$ & $9(60 \%)$ & $5(33.3 \%)$ & 0 & \\
\hline \multirow{2}{*}{ Bishop score } & $<4$ & $7(25.9 \%)$ & $11(40.7 \%)$ & $9(33.3 \%)$ & 0 & \multirow{2}{*}{$\begin{array}{l}\text { Chi square } 7.250 \\
p>0.05(0.06) \text { non- } \\
\text { significant }\end{array}$} \\
\hline & $>=4$ & $26(35.6 \%)$ & $38(52.1 \%)$ & $8(11 \%)$ & $1(1.4 \%)$ & \\
\hline \multirow{2}{*}{$\begin{array}{l}\text { Cervical } \\
\text { length }\end{array}$} & $<2.6$ & $14(27.5 \%)$ & $28(54.9 \%)$ & $8(15.7 \%)$ & $1(2 \%)$ & \multirow{2}{*}{$\begin{array}{l}\text { Chi square } 2.78 \\
p>0.05(0.427) \\
\text { non-significant }\end{array}$} \\
\hline & $>2.6$ & $19(38.8 \%)$ & $21(42.9 \%)$ & $9(18.4 \%)$ & 0 & \\
\hline
\end{tabular}

Table 2: Comparison of bishop score and cervical length with induction to delivery interval.

\begin{tabular}{|lllllll|}
\hline Group Statistics & $\begin{array}{l}\text { Induction to delivery } \\
\text { interval }\end{array}$ & $\mathbf{N}$ & Mean & $\begin{array}{l}\text { Std. } \\
\text { Deviation }\end{array}$ & $\begin{array}{l}\text { Std. Error } \\
\text { Mean }\end{array}$ & $\begin{array}{l}\text { Independent } \\
\text { t test }\end{array}$ \\
\hline \multirow{2}{*}{ Bishop score } & $>=24.00$ & 11 & 4.1818 & 1.16775 & 0.35209 & 0.926 \\
\cline { 2 - 6 } $\begin{array}{l}\text { Cervical } \\
\text { Length }\end{array}$ & $>24.00$ & 74 & 4.5676 & 1.30417 & 0.15161 & \multirow{2}{*}{$7.778^{*}$} \\
\cline { 2 - 6 }
\end{tabular}

$* \mathrm{p}<0.001$

Table 3: Correlation of the outcome measures with mean bishop score and cervical length.

\begin{tabular}{|llllll|}
\hline Descriptive Statistics & Mean & $\begin{array}{l}\text { Std. } \\
\text { Deviation }\end{array}$ & N & Pearson correlation $\mathbf{R}$ & Pearson correlation R \\
\hline interval & & 1.29 & 85 & $-0.280^{* *}$ & $\begin{array}{l}\text { Induction to delivery } \\
\text { interval }\end{array}$ \\
\hline Bishop score & 4.52 & 0.49 & 85 & $0.875^{* *}$ & $0.274^{*}$ \\
\hline Cervical Length & 2.64 & $0.86^{* *}$ & \\
\hline
\end{tabular}

*. Correlation is significant at the 0.05 level **. Correlation is significant at the 0.01 level

Table 4: Comparison of predictive values of bishop score and cervical length.

\begin{tabular}{|llllll|}
\hline Variable & Sensitivity & Specificity & PPV & NPV & Predictive value \\
\hline Bishop Score $\mathbf{Z 4}$ & $70.3 \%$ & $45.5 \%$ & $89.7 \%$ & $18.5 \%$ & 0.296 \\
\hline TVS cervical length $\mathbf{5 2 . 6}$ & $58.1 \%$ & $100 \%$ & $100 \%$ & $26.2 \%$ & $<0.001$ \\
\hline
\end{tabular}

There were $15 \%$ deliveries by caesarean section and the indication were foetal distress for $53 \%$, non-progression of labour for $40 \%$ and meconium stained liquor for $7 \%$ of the cases. Among the 74 cases delivered vaginally within 24 hours, the Mean \pm SD of the Bishop score variable was $4.1 \pm 1$ and for the cervical length $2.5 \pm 0.4$. There was a significant negative correlation between the bishop score and the induction to delivery interval $(\mathrm{p}<0.0001)$. $\mathrm{R}^{2}=0.078$ which means that only $7.8 \%$ variation was explained by the bishop score for induction to active phase delivery. There was a significant positive correlation $\mathrm{r}=0.875$ between cervical length and the Induction to delivery interval $(\mathrm{p}<0.0001)$ and $\mathrm{R}^{2}=0.765$ which means that $75.5 \%$ variation was explained by the cervical length (Table 3 ).

The cervical length appears to be a better predictor than the Bishop Score with a sensitivity of 0.703 and a 
specificity of 0.82 compared to 0.9 and 0.94 respectively. In the receiver operating characteristic curves, the best cut-off point for the prediction of successful induction was $2.6 \mathrm{~cm}$ cervical length and 4 for the Bishop score (Figure 1).

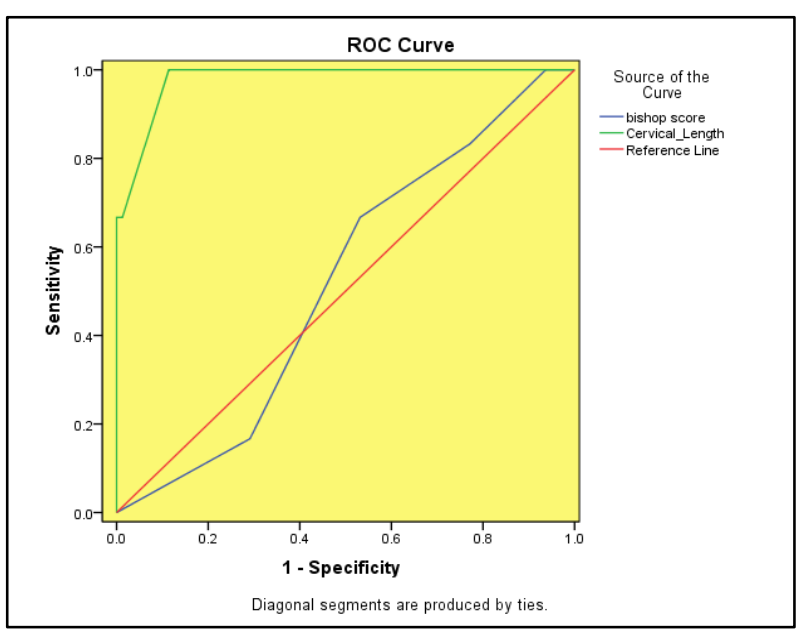

Figure 1: ROC curve for predicting successful labour induction.

Though Bishop score has more sensitivity than cervical length, specificity and positive predictive value of the trans vaginal cervical length is $100 \%$. Significant predictive value is obtained for cervical length $<0.001$. $\mathrm{P}$ value for Bishop Score is 0.296. So trans vaginal cervical length found to be better predictor of successful induction of labour in terms of delivery within 24hrs when compared to Bishop Score (Table 4).

\section{DISCUSSION}

Induction of labour is one of the common interventions in obstetric practice and is defined as the process of artificially stimulating the uterine contractions resulting in initiation of labour. The transvaginal ultrasonographic measurement of cervical length has been extensively studied in predicting the risk of preterm delivery. ${ }^{3}$ Short cervix detected by the transvaginal ultrasonogram has been associated with more incidence of preterm labour and it is proposed as a proof of the process of cervical effacement. ${ }^{4}$

The induction of labour is considered successful when it results in the initiation of labour. ${ }^{5}$ The cervical dilatation of $3-4 \mathrm{~cm}$ or greater in the presence of uterine contractions, is usually considered a reasonable threshold for diagnosis of active labour. In 1964, Bishop described a scoring system for determining a patient's suitability for elective induction of labour. ${ }^{6}$ In 1977, Harrison et al studied the antenatal women cervical assessment at 36 weeks gestation by Bishop score and again at 40 weeks gestation. The study showed a significant increase in Bishop score during the last four week of pregnancy. The study also confirmed the association between the Bishop score and the duration of labour following induction of labour. About $87 \%$ of the study population had a score of 7 or more delivered in less than 9 hours' time, whereas only $44 \%$ patients having score of 4 or less delivered within this time interval. Lange et al ${ }^{8}$ evaluated about 1,189 patients for whom induction of labour done for obstetric or medical indications and the outcome measured in terms of successful vaginal deliveries. The above study confirmed that the Bishop score, with effacement expressed as centimetres of cervical length correlated well with the likelihood of successful induction. The study concluded that cervical length was the most important factor when compared with the other factors considered in the Bishop score.

In recent studies, ultrasound assessment of preinduction cervical assessment has been evaluated. O'Leary and Ferrell proposed a 'semi quantitative' ultrasound scoring system and evaluated this system in comparison with the modified Bishop score. ${ }^{9}$ The study was conducted using transabdominal ultrasound to evaluate the thickness and contour of the lower uterine segment, the length and dilation of the cervix, and the station of the presenting part. The authors found that the ultrasound scoring system correlated well with the modified Bishop score. They concluded that both digital or ultrasound assessment was associated with a high likelihood of successful induction.

However, some of the studies on the evaluation of pre induction sonographic measurement of cervical length have shown conflicting results. Paterson Brown et al evaluated about 50 pregnancies before induction and reported that, although the Bishop Score correlated significantly with successful vaginal delivery, the score was not a satisfactory predictor of successful induction. ${ }^{10}$ They also concluded that ultrasonographically measured cervical length was no comparable with the Bishop Score and there was no significant shorter duration of the induction to delivery interval. Boozarjomehri et al evaluated 53 women before induction and reported that the ultrasonographically measured cervical length correlated with the duration of the latent phase of the labour but there was no significant association with the induction to delivery interval and to cervical effacement measured by digital examination. ${ }^{11}$

Watson et al evaluted 109 women before induction and reported a significant association between ultrasonographically measured cervical length and clinical assessment of cervical effacement, but both the methods was not useful in predicting the length of the latent phase of labour. ${ }^{12}$ Gonne et al evaluated 86 women before induction and reported significant association between both the Bishop Score and sonographically measured cervical length with successful vaginal deliveries and shorter induction to delivery interval. ${ }^{13}$ Ware and Raynor evaluated 77 
women before induction and found that both sonographically measured cervical length and Bishop score predicted induction-to-delivery interval and increased likelihood of successful vaginal delivery. ${ }^{14}$ Pandis et al studied about 240 women with singleton pregnancies at 37-42wks of gestation for the prediction of successful vaginal deliveries following cervical length assessment with ultrasonogram. ${ }^{15}$ The study reported that vaginal delivery occurred in $194(80.8 \%)$ women and among them, about $142(73.2 \%)$ delivered within $24 \mathrm{hrs}$ of induction. The study also concluded that cervical length by ultrasound was a better method of assessing the cervical length than Bishop Score to predict vaginal delivery within $24 \mathrm{hrs}$ of induction.

In our study we defined successful induction of labour as vaginal delivery occurring within $24 \mathrm{hrs}$ and has demonstrated that, in primi singleton pregnancies undergoing induction of labour with dinoprostone gel at 37-42 wks., successful vaginal delivery within $24 \mathrm{hrs}$ of induction occurred in approximately $74 \%$. The study has also demonstrated that induction to delivery interval is significantly associated with both the preinduction bishop score and the ultrasonographically measured cervical length, higher the Bishop score and lesser the cervical length better the likelihood of vaginal delivery. TVS cervical length was a better predictor of successful labour induction in terms of delivery within $24 \mathrm{hrs}$ of induction .Both sonographic cervical assessment and the Bishop Score successfully predicted vaginal delivery within $24 \mathrm{hrs}$. As the cervical length increases the likelihood of delivering within $24 \mathrm{hrs}$ decreases and as bishop score increase, the likelihood of delivering within $24 \mathrm{hrs}$ increases. However, the receiver operating characteristic curves for the two variables showed that, the sensitivity of ultrasonographically measured cervical length in predicting successful induction of labour was higher than that for the Bishop Score. ROC curves were constructed to determine appropriate cut off for bishop score and trans vaginal cervical length in predicting the labour induction have shown that 4 is the best cut off for Bishop Score and 2.6 is for trans vaginal cervical length. In our study though the sensitivity of the Bishop Score in predicting the successful labour induction is higher $(70.3 \%)$ compared with that of cervical length measured trans vaginally $(58.1 \%)$ the specificity and positive predictive value for the cervical length was $100 \%$ compared with the Bishop Score $(45.5 \%$ and $89.7 \%$ are respectively). About $67 \%$ of the long cervix group $(3.2-5 \mathrm{~cm})$ remained undelivered after $24 \mathrm{hrs}$ compared to $33 \%$ of women in the low Bishop Score group. This shows that ultrasonographic cervical length measurement is a better method of cervical assessment than the Bishop Score for predicting successful induction of labour.

Transvaginal sonographic measurement of cervical length requires expertise and provides a useful prediction of the likelihood of vaginal delivery within $24 \mathrm{hrs}$ of induction and of the induction to delivery interval. It helps in counselling the women regarding the outcome of labour induction. Women with a cervical length of less than 2.6 $\mathrm{cm}$ can be counselled that delivery will possibly occur within $24 \mathrm{hrs}$ of induction, whereas those with cervical length of $3 \mathrm{~cm}$ can be advised that they have an approximately $67 \%$ chance of remaining undelivered after this interval. Bishop score remains a useful test in the setting where the equipment and experts are not available as it is a simple, inexpensive test and does not require a technical expert.

\section{CONCLUSION}

Bishop score and transvaginal cervical length both are good predictors of successful induction of labour resulting in vaginal deliveries. Transvaginal cervical length provides a better prediction of the likelihood of vaginal delivery within $24 \mathrm{hrs}$ of induction. The transvaginal ultrasonographic measurement of cervical length could be used as a better alternative to Bishop Score for successful labour induction in the setting where the appropriate equipment and expertise are available.

Funding: No funding sources

Conflict of interest: None declared

Ethical approval: The study was approved by the Institutional Ethics Committee

\section{REFERENCES}

1. RCOG. Induction of labour. In Evidence based Clinical Guideline Number 9 London:2001.

2. Bishop EH. Pelvic scoring for elective induction. 1964;24:266-8.

3. Iams JD. The length of the cervix and the risk of spontaneous premature delivery. National Institute Of Child Health and Human development Maternal Fetal Medicine Units Network.1996;334:567-72.

4. Vonda Ware. Transvaginal Ultrasonographic cervical measurement as a predictor of successful labor induction.182(5)2000:1030-2.

5. Cunningham FG. Induction and augmentation of labor. 21st Ed. New York, Ny: MC Graw-Hill; 2001:469-81.

6. Bishop EH. Pelvic scoring for elective induction. 1964;24:266- 8.

7. Harrison RF, Flynn M, Craft I. Assessment of factors constituting an inducibility profile. 1977;49: 270.

8. Lange AP. Pre labor evaluation of pre induction scoring systems. 1982;60:137.

9. O Leary J, Ferrel RE. Comparison of Ultrasonographic and digital cervical evaluation. 1986;69:718-9.

10. Peterson Brown S, Fisk NM, Edmonds DK, Rodeck $\mathrm{CH}$. Preinduction cervical assessment by Bishop score and transvaginal ultrasound. Eur J Obstst Gynecol Reprod Biol. 1991;40:17-23.

11. Boozarjomehri F, Timor-Tritsch I, Chao CR, Fox HE. Transvaginal ultrasonographic evaluation of the 
cervix before labor: presence of cervical wedging is associated with shorter duration of induced labor. Am J Obstet Gynecol. 1994;171:1081-7.

12. Watson WJ, Stevens D, Welter S, Day D. Factors predicting successful labor induction. Obstst Gynecol 1996;88:990-2.

13. Gonen R, Degani S, Ron A. prediction of successful induction of labor. Comparison of transvaginal ultrasonography and the Bishop Score. Eur J Ultrasound. 1998;7:183-7.

14. Ware V, Raynor D. Transvaginal ultrasonographic cervical measurement as a predictor of successful labor induction. Am J Obstet Gynecol. 2000;182:1030-2.

15. Pandis. Preinduction sonographic measurement of cervical length in the prediction of successful induction of labor. Ultrasound Obstet Gynecol. 2001;623-8.

16. Rozenberg P. Comparison of digital and ultrasonographic examination of the cervix in predicting time interval from induction to delivery in women with a low bishop score. BJOG. 2005;112:192-6.

Cite this article as: Balaji TE, Vijaya S. A prospective study on comparison of cervical length measured transvaginally and bishop score in predicting successful labour induction. Int J Reprod Contracept Obstet Gynecol 2021;10:1457-62. 